\title{
Cell phone radiations and its effects in public health- comparative review study
}

\begin{abstract}
The review article compares and contrasts the existing research studies on cell phone radiations and its effects. Cell phone use has increased in recent decade and its effects needs to be reviewed. This study serves as a potential source, which can help us determine public health as well as environmental issues. The literature review observes several determinants of effects caused by cell phone radiations. The effect associated is good as well as bad. Future studies can help us optimize the determinants of cell phone radiations on human body as well as environment. It can help us prevent the hazardous effect in advance by finding the solutions.
\end{abstract}

Volume 7 Issue I - 2018

\author{
Naiya Patel \\ Long Island University, USA
}

Correspondence: Naiya Patel, Graduate Research Assistant, Long Island University, Brooklyn, New York, USA, Tel \#929-2807280, Email naiya.patel20I4@gmail.com

Received: November 14, 2017| Published: January 24, 2018

Keywords: cell phone radiations, effects of radiations, public health, environment

\section{Introduction}

Today in the $21^{\text {st }}$-century era we are living a life in which technology has taken control over our physical caliber be it computers, laptops, the navigation system, motor vehicles, radar, satellite or be it minutest things like cell phone or Wi-Fi modem. Each of us is daily users of one or the other electronic gadgets depending on our choice and gets attracted towards it without considering any side effects. As the phrase says it all, "Everything which glitters is not gold." In our day to day busy life, we are concerned with ease of performing a task over fingertips which would take micro-milliseconds rather than spend a couple of hours be it texting or voicemail. This is the major reason why we cannot see the invisible harmful effects of radiations emitted through these gadgets to environment and its effect on us.

In this current review study, we are considering one such electronic gadget which is cell phone. It is one of those electronics which none us are unaware about. Cell phones make our lives easy in terms of responding someone quickly, passing on the message, recording our heart rates, monitoring our health, checking time, recording something, sharing pictures and what not. You name it and it will be able to perform depending on the cell phone model you are using.

As per an article by Sue Kovach in Life extension magazine, the cell phones reach the market without safety testing. Cell phone came into existence in early 1980's, when technology used for the defense was made open for commercial use in terms of communication. The major big companies with the intention of making profit pressurized the government including Food and Drug Administration (FDA) to make cell phones available in the market to be sold without pre-market testing. The category which excluded cell phones from pre-market testing was "low power exclusion". It means that cell phones are not as dangerous or hazardous as other gadgets which emit microwaves or radiations like microwave ovens. As per National Cancer Institute NIH, there exist three major reasons why people have started considering harmful effects of cell phone usage. Firstly cell phones emit nonionizing radiations which the tissues can absorb, secondly, as of December 2014; there were more than 327.5million cell phone users in United States itself. Also, as per reports by Telecommunications union, globally the figure of cell phone subscribers is 5 billion. Lastly, eventually, the number of call length per day, the amount of texting and the amount of time spent near cell phones has increased.
The question now arise is, what health effects do the radiofrequency energy has over us? What can be the potential public health effects of this tiny gadget on us and the environment in general? The gadget surely emits certain radiations in the surroundings which is radiofrequency energy. It is a type of electromagnetic radiation which can be categorized as ionizing and non-ionizing radiations. The only health effect which is proven through studies is heating up of biological tissue near which cell phones are held. The following paragraphs will be discussing about few important studies performed in the field which will help us get a better idea in terms of effects of using cell phone. Each study is reviewed and contrasted with the other. At the end we have summarized the contrasting features of each study (Figure 1).

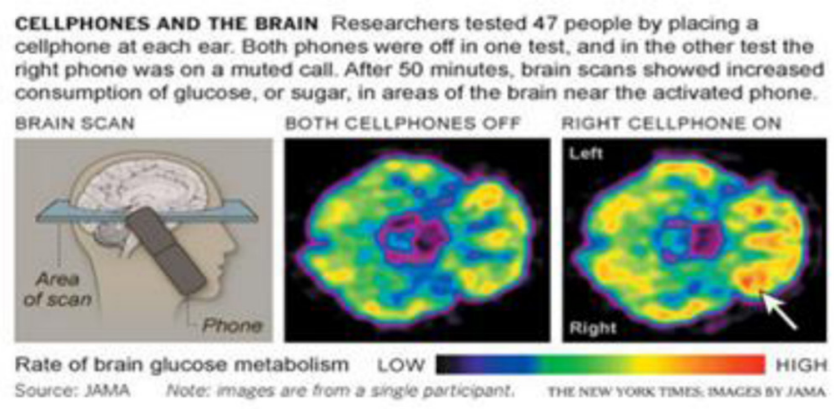

Figure I The figure retrieved from JAMA reflects the effect of cell phone radiation on brain tissue.

\section{A Review of research studies}

i. The study by Acharya et al. ${ }^{1}$ focuses on both the behavioral aspect of the adverse effect of prolonged cell phone use rather than just insomnia and headaches. Cell phone use is extensive among college goers. Hence being not aware about the hazardous effects of cell phone radiations over human body is dangerous. The study reflects an association between extensive cell phone usage and irritability along with other psychological changes like lack of concentration. Several measures have been suggested to avoid extensive use of cell phone by the author. The study methods used is a pre-tested questionnaire. The results are reliable as the questionnaire was 
pretested and it is a retrospective study so that way we can get an idea of already exposed cases and their association with adverse psychological effects. The strength of the study was sample size as well as age of below 20years. It is the most vulnerable population which is undergoing brain and neuronal development. The weakness is the analysis tool used for the study. It is not clear through his paper that did the use Pearson's rho or other paired t-tests in order to confirm the association between variables. Future studies need to done using the powerful statistical tool as well as study design i.e. quasi-experimental study etc.

ii. The research article by Li et al. ${ }^{2}$ talks about effect of electromagnetic radiation over brain activity. Cell phone use releases electromagnetic radiations. The foundation of brain activity is the neuronal cells The neuronal activity is affected by thermal effect caused due to extensive use of cell phones. Several brain dysfunctions are associated due to extensive use of cell phones like Parkinson's disease or Alzheimer's disease as well as lack of concentration etc. The research reflects the results of association between electromagnetic radiation as well as hindrance in firing activity of neuronal cells. The most affected cells are information processing neuronal network. The current research study by Li et al. ${ }^{2}$ uses a mathematical model to describe the effect of electromagnetic radiation on neuronal firing activity by introducing an additional membrane current into the Hodgkin-Huxley neuron model. The results are valid as they are using a mathematical model. In the energy absorption assumption, Wachtel et al. predicted that a part of the power of electromagnetic radiation could be absorbed by the neuronal media and this absorbed power should be translated into an additional polarizing current in neuronal media. This would thus be consistent with the suppression effect of electromagnetic radiation on neuronal firing which the current research study is using. The strength of the study is the mathematical multi-stage model while the weakness is that there are no proven facts that the same applies universally as electromagnetic radiation distribution differs in different neuronal networks. Future studies should take into consideration the electromagnetic field distribution in different neuronal network setup.

iii. The research by Thomas et al. ${ }^{3}$ describes the effect of cell phone usage and its association with cognitive function disruption over children and adolescent. Many studies have been performed on the same topic but what makes it special is the study focuses on children and adolescent who are in the growing phase of their life which also includes growth and development of neuronal cells as well as the brain. Changes in cognitive function were associated with increase in exposure of SMS and voice calls over a latency period. Socio-demographic data was collected as well as Cognitive functions were assessed with a computerized test battery and the Stroop Color-Word test. Hence the results are valid and reliable. The strength of the study is statistical analysis method used while the weakness is reliability on self-report by the participants which might give rise to bias. The study is first of its kind which finds an association between delay in response and cell phone usage rather than accuracy in terms of cognitive functioning. But future such studies should not collect the data which are self-reported but rather perform data collection method which would not include self-reporting. One such design is quasi-experimental study or retrospective study. iv. Cell phones are in trend be it in class, work or emergency. Youngsters are more susceptible to adverse effect of extensive use of cell phones compared to other population groups. The current study by Zarghami et al. ${ }^{4}$ assesses the extent to which students of Mazandaran university of medical sciences operate as well as use cell phones and determines the health effect of cell phone use them after lights-out like a headache, tiredness, insomnia as well as the lack of ability to focus. The results reflect the association between extensive use of cell phone and lack of ability to concentrate, insomnia as well as a head ache. The methods used by the author in his cross sectional study is apt and the results are reliable as the author used logistic regression and chi-square test. The strength of the study is the statistical analysis method used while the weakness is small sample size which may not represent the population worldwide. The collected data was self-reported hence giving chance to biases in the results. The consideration to avoid biases in future such studies is a way to get valid results or perform different study design.

v. Contrasting study-The study by Movvahedi et al. ${ }^{5}$ is the first contrasting study to my knowledge which reflects a beneficial effect of short-term RF radiation exposure to improvement in short-term memory in kids and children. Mobile phone emitted radiations have proven to reduce reaction time as well as enhance short-term memory thereby opening doors for future cognitive impairment therapy in kids as well as adults. The study is performed among 7 to 10years of aged children of boy's elementary school in Iran. The results of the study seem to be valid as they are using a way to test both real and sham exposures in order to avoid discrepancies. A VRT, as well as short-term memory test, is performed to which students are oriented before the test. It was a double-blinded study which in way is strength of the study as it has valid results without biases. The weakness is, the researchers didn't consider different hemispheres of brain and the effect of radiations over them (Figure 2). In future studies researchers might want to consider the association of radiations with different hemisphere of brain involved in different social as well as cognitive functions (Table 1).

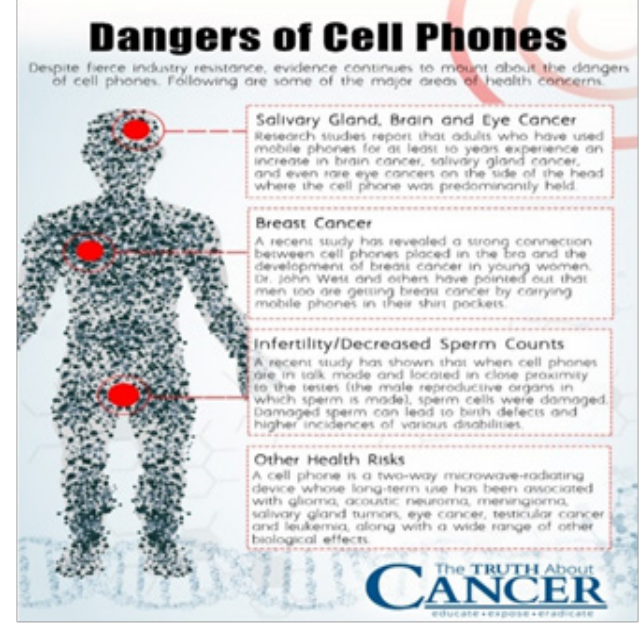

Figure 2 The figure retrieved from the truth about cancer website reveals potential effects of cell phone usage. It has potential cancerous effects on several body tissues. 
Table I Summary of contrasting features for studies.

Studies for Cell phone use ${ }^{5}$
Studies against Cell phone use ${ }^{2}$

\section{Method}

Computer-assisted VRT test was taken and it was tested in both A mathematical model was used to describe the effect of electromagnetic sham and real time exposure radiation on neuronal firing activity by introducing an additional membrane current into the Hodgkin-Huxley neuron model.

\section{Results}

Improve short term memory as well as no delay in response It shows association between short-term acute radiation exposure and no changes in brain activity
More than the thermal effect the electromagnetic power absorption causes changes in neuronal activity Suppression of neuronal activity leads to change in normal brain functioning like delay in information processing and alertness.

Flaw

They fail to consider association between different hemispheres They have not considered the distribution of electromagnetic radiations of brain like the right and left hemisphere separately and their and its effect over different neuronal networks as it differs from person to association with cell phone radiation exposure person.

\section{Discussion}

Hence one thing is clear that the electromagnetic radiations, be it ionizing or non-ionizing have the potential greatest effect on developing cells or cells who have the ability to regenerate/divide and reproduce. Neuronal cells of the population below age 20years are more prone to bad or good effects of cell phone radiations as compared to elder adults when exposed. Be it bad or good, cell phones radiations have some potential effects on us. As they say anything in excess is poison. The radiations from cell phone tower or base station as well as from personal phones cause harmful effects to all mammals. If used for the limited amount of time over a day can be the only solution to avoid excess exposure and its harmful health effects. ${ }^{6-8}$ Cell phone radiation effects can be determined by several factors which are as follows:

\section{Determinants of cell phone radiations effect}

i. Age- Population in a developmental stage of their life are more prone to cancerous or harmful effect of Radiations

ii. Genetics- Different species as well as single species but with different genetics react differently to similar radiation exposure

iii. Gender- Female is more prone to aggravated inflammatory reaction to an exposure as compared to their male counterparts

iv. Duration of exposure- The longer the exposure more harmful is the effect

v. Dose/intensity/amount of radiation during exposure- More the intensity greater is the harmful effect of the exposure to the susceptible

vi. Way of talking/handling cell phone while using- Rather than putting cell phone near ears if we put it on speaker mode and talk, it will have less exposure effect to the user. Texting makes us less exposed to the radiation intensity as compared to calling. Also keeping cell phones away from us during sleep or at home makes us less exposed to the radiations.
As per American Cancer Society, there are several suggestions made in order to reduce radio frequency exposure emitted by cell phones. Some of it is:

a. Use of speaker mode while talking over cell phone or use of headphone/earpiece which can be both corded or cordless

b. Texting over talking

c. Avoid frequent use of cell phone, all day long. If given an option of conventional phone, avoid use of cell phones

d. Choice of lower SAR (Specific Absorption Rate) valued cell phone

Hence, the review article contributes in advancing the field of public health research. It opens the door for future studies which can take into consideration the determinants of cell phone radiation effects. It will help make the foundations strong and help us achieve better translational results into real world (Figure 3).

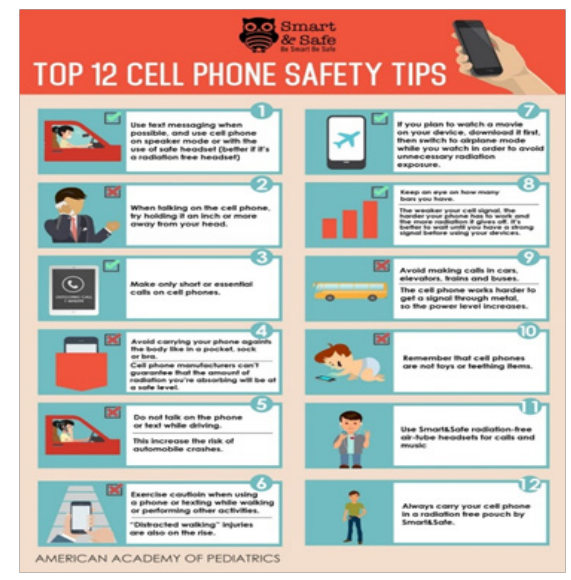

Figure 3 Retrieved from American Academy of Pediatrics. It mentions I 2 cell phone safety tips. 


\section{Conflict of interest}

None

\section{Acknowledgement}

Jolanta Kruszelnicka, Environmental and Occupational Health Educator Adjunct Assistant Professor, Long Island University, Brooklyn, NY. Dr. Bojana Beric, Chair of the Department of Public Health, Long Island University, NY.

\section{Funding}

None.

\section{References}

1. Acharya JP, Acharya I, Waghrey D. A study on some psychological health effects of cell-phone usage amongst college going students. IJMRHS 2013;2(3):388-394.
2. Li J, Liu S, Liu W, et al. Suppression of firing activities in neuron and neurons of network induced by electromagnetic radiation. Nonlinear Dynamics. 2016;83(1-2):801-810.

3. Thomas S, Benke G, Dimitriadis C, et al. Use of mobile phones and changes in cognitive function in adolescents. Occupational and environmental medicine. 2010;67(12)

4. Zarghami M, Khalilian A, Setareh J, et al. The Impact of Using Cell Phones After Light-Out on Sleep Quality, Headache, Tiredness, and Distractibility Among Students of a University in North of Iran. Iran J Psychiatry Behav Sci. 2015;9(4):e2010.

5. Movvahedi MM, Golpayegani TA, Mortazavi SAR, et al. Does exposure to GSM $900 \mathrm{MHz}$ mobile phone radiation affect short-term memory of elementary school students?. J pediatr neurosci. 2014;9(2):121.

6. What causes cancer? American Cancer Society. 2018.

7. Cell Phones and cancer risk. NIH, National Cancer Institute. 2016.

8. Sue Kovach.The Hidden Dangers of Cell Phone Radiation. Life extension magazine. 2017. 